\title{
BitTorrent Swarm Analysis through Automation and Enhanced Logging
}

\author{
Răzvan Deaconescu, Marius Sandu-Popa, Adriana Drăghici, Nicolae Tăpus, \\ Automatic Control and Computers Faculty \\ University Politehnica of Bucharest \\ Bucharest, 060042 \\ Email: \{razvan.deaconescu, nicolae.tapus\}@cs.pub.ro, \{marius.sandu- \\ popa, adriana.draghici\} @cti.pub.ro
}

\begin{abstract}
Peer-to-Peer protocols currently form the most heavily used protocol class in the Internet, with BitTorrent, the most popular protocol for content distribution, as its flagship.A high number of studies and investigations have been undertaken to measure, analyse and improve the inner workings of the BitTorrent protocol. Approaches such as tracker message analysis, network probing and packet sniffing have been deployed to understand and enhance BitTorrent's internal behaviour. In this paper we present a novel approach that aims to collect, process and analyse large amounts of local peer information in BitTorrent swarms. We classify the information as periodic status information able to be monitored in real time and as verbose logging information to be used for subsequent analysis. We have designed and implemented a retrieval, storage and presentation infrastructure that enables easy analysis of BitTorrent protocol internals. Our approach can be employed both as a comparison tool, as well as a measurement system of how network characteristics and protocol implementation influence the overall BitTorrent swarm performance. We base our approach on a framework that allows easy swarm creation and control for different BitTorrent clients. With the help of a virtualized infrastructure and a client-server software layer we are able to create, command and manage large sized BitTorrent swarms. The framework allows a user to run, schedule, start, stop clients within a swarm and collect information regarding their behavior.
\end{abstract}

Keywords - BitTorrent; swarm analysis; protocol messages;logging

\section{INTRODUCTION}

With the exponential growth of digital content and available information, Peer-to-Peer systems have become the most important protocol class for data distribution [7].

Among the wide variety of Peer-to-Peer protocols (Kazaa, DirectConnect, eDonkey, Kademlia, Gnutella), the BitTorrent protocol has proven to be the nowadays "killer protocol". With over $30 \%$ of the Internet traffic [7], BitTorrent is the most heavily used protocol in the Internet. The use of simple yet powerful techniques such as tit-for-tat or rarest-piece-first have selected BitTorrent as the best choice for large data distribution.

In order to keep up with recent advances in Internet technology,streaming and content distribution, Peer-to-Peer systems(and BitTorrent) have to adapt and develop new, attractive and useful features. Extensive measurements, coupled with carefully crafted scenarios and dissemination are important for discovering the weak/strong spots in Peer-to-Peer based data distribution and ensuring efficient transfer.

In this paper we present a framework for running, commanding and managing BitTorrent swarms. The purpose is to have access to a easy-to-use system for deploying simple to complex scenarios, make extensive measurements and collect and analyze swarm information (such as protocol messages, transfer speed, connected peers) [12]. 
International Journal of Computer Networks \& Communications (IJCNC) Vol.3, No.1, January 2011

\section{A. BitTorrent Keywords}

The heart of the BitTorrent protocol is a torrent file. The torrent file is a meta-information file containing information regarding the content to be shared/distributed. Any participant (peer) has to have access to the torrent file.

An initial peer needs to have access to the complete file for bootstrapping the transfer. This peer is called the initial seeder. A peer that has access to the complete content and it's only uploading it is called a seeder. A peer who is downloading and uploading and has incomplete access to the file, is called a leecher.

A collection of peers (seeder or leechers) who are participating in a transfer based on torrent file forms a swarm

The core of the BitTorrent protocol is the tit for tat mechanism, also called optimistic unchoking allowing for upload bandwidth to be exchanged for download bandwidth. A peer is hoping another peer will provide data, but in case this peer doesn't upload, it will be choked. Another important mechanism for BitTorrent is rarest piece first allowing rapid distribution of content across peers. If a piece of the content is owned by a small group of peers it will be rapidly requested in order to increase its availability and, thus, the overall swarm speed and performance.

\section{B. Swarm Management Framework}

The swarm management framework is a service-based infrastructure that allows easy configuration and commanding of BitTorrent clients on a variety of systems. A client application (commander) is used to send commands/requests to all stations running a particular BitTorrent client. Each station runs a dedicated service that interprets the requests and manages the local BitTorrent client accordingly.

The framework is designed to be as flexible and expandable as possible. As of this point it allows running/testing a variety of scenarios and swarms. Based on the interest of the one designing and running the scenario, one may configure the BitTorrent client implementation for a particular station, alter the churn rate by configuring entry/exit times in the swarm, add rate limiting constraints, alter swarm size, file size etc. Its high reconfigurability allows one to run relevant scenarios and collect important information to be analyzed and disseminated.

Through automation and client instrumentation the management framework allows rapid collection of status and logging information from BitTorrent clients. The major advantages of the framework are:

- automation - user interaction is only required for starting the clients and investigating their current state;

- complete control - the swarm management framework allows the user/experimenter to specify swarm and client characteristics and to define the context/environment where the scenario is deployed;

- full client information - instrumented clients output detailed information regarding the inner protocol implementation and transfer evolution; information are gathered from all client and used for subsequent analysis.

\section{Information collection}

Based on the infrastructure we present a novel approach involving client-side information collection regarding client and protocol implementation. We have instrumented a libtorrentrasterbar client [2] and a Tribler [8] client to provide verbose information regarding BitTorrent protocol implementation. These results are collected (see Section VII) and subsequently processed and analysed through a rendering interface (see Section VIII).

Swarm measured data are usually collected from trackers. While this offers a global view of the swarm it has little information about client-centric properties such as protocol implementation, neighbour set, 
International Journal of Computer Networks \& Communications (IJCNC) Vol.3, No.1, January 2011

number of connected peers, etc. A more thorough approach has been presented by Iosup et al. [15], using network probes to interrogate various clients.

Our approach, while not as scalable as the above mentioned one, aims to collect client-centric data, store and analyse it in order to provide information on the impact of network topology, protocol implementation and peer characteristics. Our infrastructure provides micro-analysis, rather than macroanalysis of a given swarm. We focus on detailed peer-centric properties, rather than less-detailed global, tracker-centric information. The data provided by controlled instrumented peers in a given swarm is retrieved, parsed and stored for subsequent analysis. Section III details the modules and information flow in our infrastructure.

We differentiate between two kinds of BitTorrent messages, thoroughly described in Section VI: status messages, which clients provide periodically to report the current session's download state, and verbose messages that contain protocol messages exchanged between peers (chokes, unchokes, peer connections, pieces transfer etc.).

As BitTorrent clients for our experiments, we chose the libtorrent-rasterbar [2] implementation and Tribler [8]. In our studies [10], libtorrent-rasterbar has proven to be the fastest BitTorrent client, while Tribler is one of the most feature rich client from a scientific point of view. Each client outputs information in a specific format such that a different message parser is required for each client. Detailed information on the messages and client instrumentation are presented in Section VI.

Depending on the level of control of the swarm, we define two types of environments. A controlled environment, or internal swarm uses only instrumented controlled clients. We have complete control over the network infrastructure and peers. A free environment or external swarm is usually created outside the infrastructure, and consists of a larger number of peers, some of which are the instrumented controlled clients. Our experiments so far have focused on controlled environments; we aim to extend our investigations to free environment swarms.

\section{P2P-Next}

This paper is part of the research efforts within the P2P -Next FP7 project [3].

\section{CONTEXT}

The proposed swarm management framework was created and designed to provide data for the BitTorrent analysis system presented in [12]. This system is focused on offering the means to collect, store and visualize BitTorrent swarm data at a peer-centric level. This degree of detail is provided at BitTorrent client level, thus our experiments aim to gather information about protocol implementation and peer characteristics.

The framework supports experiments on instrumented Bit- Torrent clients (currently only Tribler [8] and Hrktorrent [6] (based on libtorrent-rasterbar)), which provide the data needed for the analysis system. These clients run in command-line mode and are configured to output the communication between peers at a protocol level.

The analysis system consists of parsers and a rendering engine that interact with a relational database. The messages exchanged between peers and those output by the client with the state of the transfers, are stored in verbose logs files and status log files. The parsers take these files as input, in order to extract the information provided by each message and store it into the database. The analysis of protocol messages coupled with the information regarding the transfer status allows detection of weak spots of the protocol implementation, thus providing feedback about the client or possible improvements.

\section{ARCHITECTURE}

The software service infrastructure was designed with the goal of remotely controlling BitTorrent clients. Its architecture ( Fig. 1) is built on a client-server model, with a single client addressed as Commander and multiple servers. The BitTorrent clients reside in OpenVZ virtual containers and are 
controlled only through the Server service, by interacting with the Commander interface. A SSH connection is used by the Commander for the initial bootstrapping, in case the service is not active.

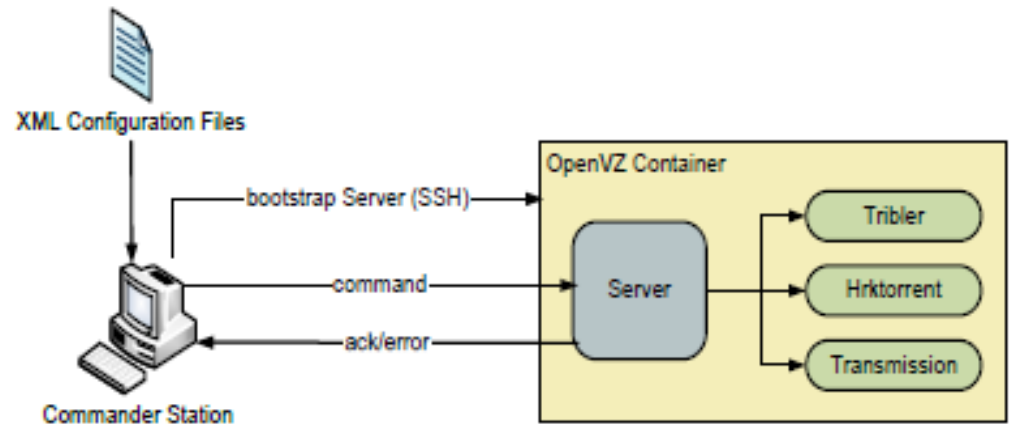

Figure 1. Software Service System overview

The services are completely implemented in Python, easily allowing extensions and offering improved maintainability over the shell scripts used in an earlier virtualized testing environment [11].

The BitTorrent scenarios are defined using XML configuration files which can be considered as input to the Commander. These files contain information not only about each container that should be used, but also about the torrent transfers, like file names and paths. A more through description can be found in section IV-B.

In order to examine BitTorrent transfer at a protocol implementation level, we propose a system for storing and analyzing logging data output by BitTorrent clients. It currently offers support for hrktorrent/libtorrent [6] [2] and Tribler [8].

Data is provided by BitTorrent clients in log files that are parsed, stored, intepreted and rendered. We have divided the information generated by clients into status log files and verbose log files, each composed of one of two types of messages.

Status messages are periodic messages reporting session state. Messages are usually output by clients at every second with updated information regarding number of connected peers, current download speed, upload speed, estimated time of arrival, download percentage, etc. Status messages are to be used for real time analysis of peer behaviour as they are lightweight and periodically output (usually every second). Verbose messages or log messages provide a thorough inspection of a client's implementation. The output is usually of large quantity (hundreds of MB per client for a one-day session). Verbose information is stored in client side log files and is subsequently parsed and stored.

Currently, the infrastructure consists of the following modules:

- Parsers - receive $\log$ files provided by BitTorrent clients during file transfers. Due to differences between log file formats, there are separate pairs of parsers for each client. Each pair analyses status and verbose messages.

- Database Access - a thin layer between the database system and other modules. Provides support for storing messages, updating and reading them.

- SQLite Database - contains a database schema with tables designed for storing protocol messages content andpeer information.

- Rendering Engine - consists of a GUI application that processes the information stored in the database andrenders it using plots and other graphical tools. 


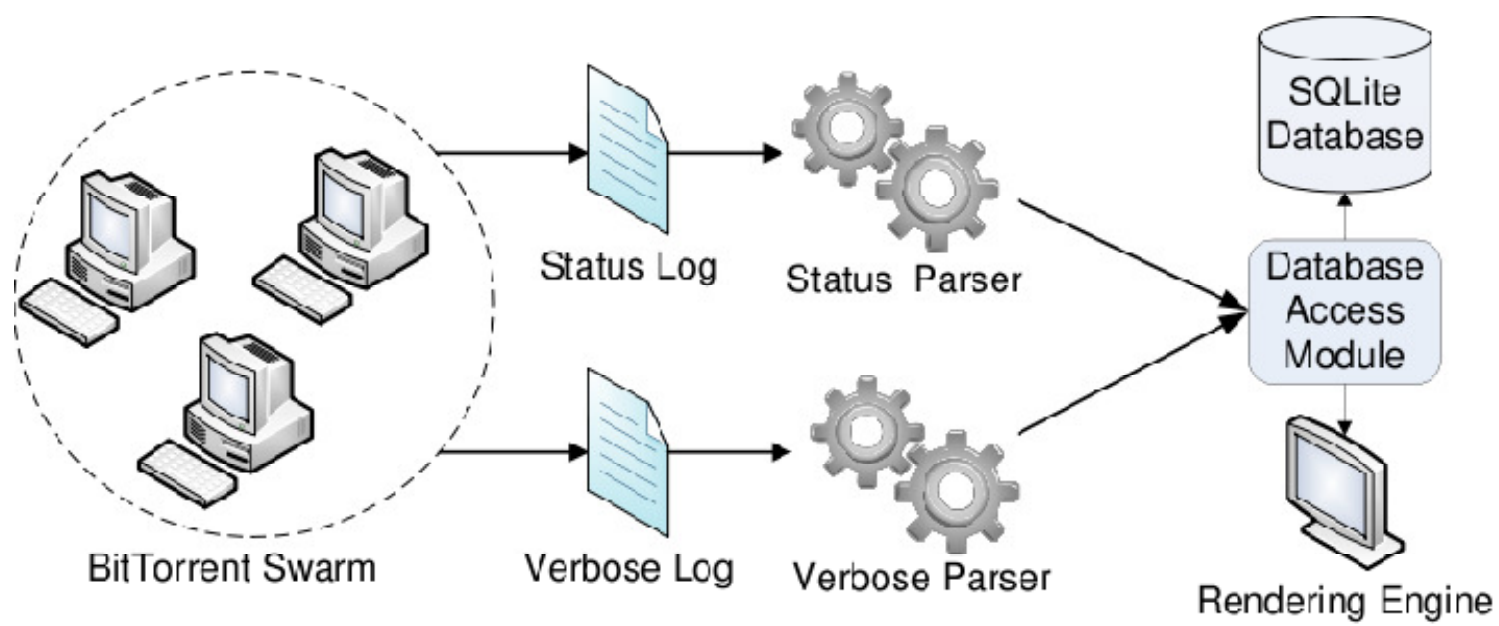

Figure 2. Logging system overview

As shown in figure 4, using parsers specific to each type of logging file, messages are sent as input to the Database Access module that stores them into an SQLite database. In order to analyse peer behaviour the Rendering Engine reads stored logging data using the Database Access module and outputs it to a graphical user interface. More information on each component is presented in the following section.

\section{ARCHITECTURE}

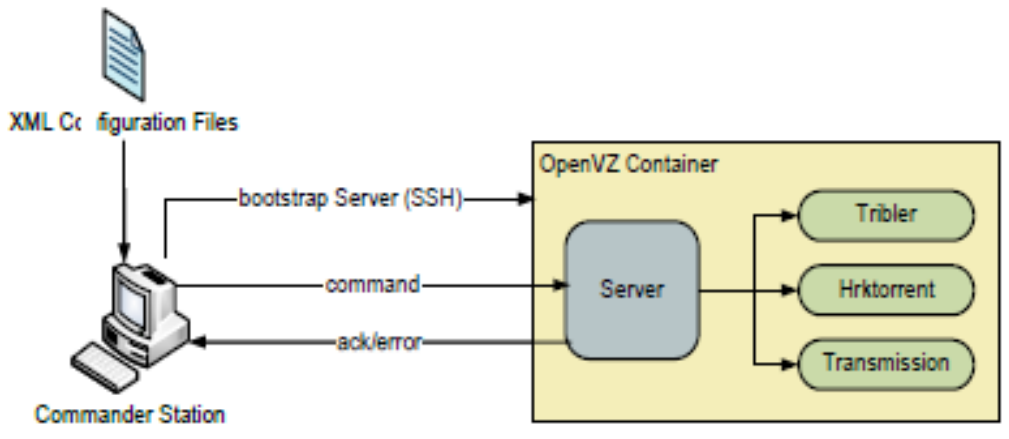

Figure 3. Software Service System overview

The software service infrastructure was designed with the goal of remotely controlling BitTorrent clients. Its architecture(Fig. 1) is built on a client-server model, with a single client addressed as Commander and multiple servers. The BitTorrent clients reside in OpenVZ virtual containers and are controlled only through the Server service, by interacting with the Commander interface. A SSH connection is used by the Commander for the initial bootstrapping, in case the service is not active.

The services are completely implemented in Python, easily allowing extensions and offering improved maintainability over the shell scripts used in an earlier virtualized testing environment [11].

The BitTorrent scenarios are defined using XML configuration files which can be considered as input to the Commander. These files contain information not only about each container that should be used, but also about the torrent transfers, like file names and paths. A more through description can be found in section IV-B. 
In order to examine BitTorrent transfer at a protocol implementation level, we propose a system for storing and analyzing logging data output by BitTorrent clients. It currently offers support for hrktorrent/libtorrent [6] [2] and Tribler [8].

Data is provided by BitTorrent clients in log files that are parsed, stored, intepreted and rendered. We have divided the information generated by clients into status log files and verbose log files, each composed of one of two types of messages.

Status messages are periodic messages reporting session state. Messages are usually output by clients at every second with updated information regarding number of connected peers, current download speed, upload speed, estimated time of arrival, download percentage, etc. Status messages are to be used for real time analysis of peer behaviour as they are lightweight and periodically output (usually every second). Verbose messages or log messages provide a thorough inspection of a client's implementation. The output is usually of large quantity (hundreds of MB per client for a one-day session). Verbose information is stored in client side log files and is subsequently parsed and stored.

Currently, the infrastructure consists of the following modules:

- $\quad$ Parsers - receive log files provided by BitTorrent clients during file transfers. Due to differences between log file formats, there are separate pairs of parsers for each client. Each pair analyses status and verbose messages.

- Database Access - a thin layer between the database system and other modules. Provides support for storing messages, updating and reading them.

- SQLite Database - contains a database schema with tables designed for storing protocol messages content and peer information.

- Rendering Engine - consists of a GUI application that processes the information stored in the database andrenders it using plots and other graphical tools.

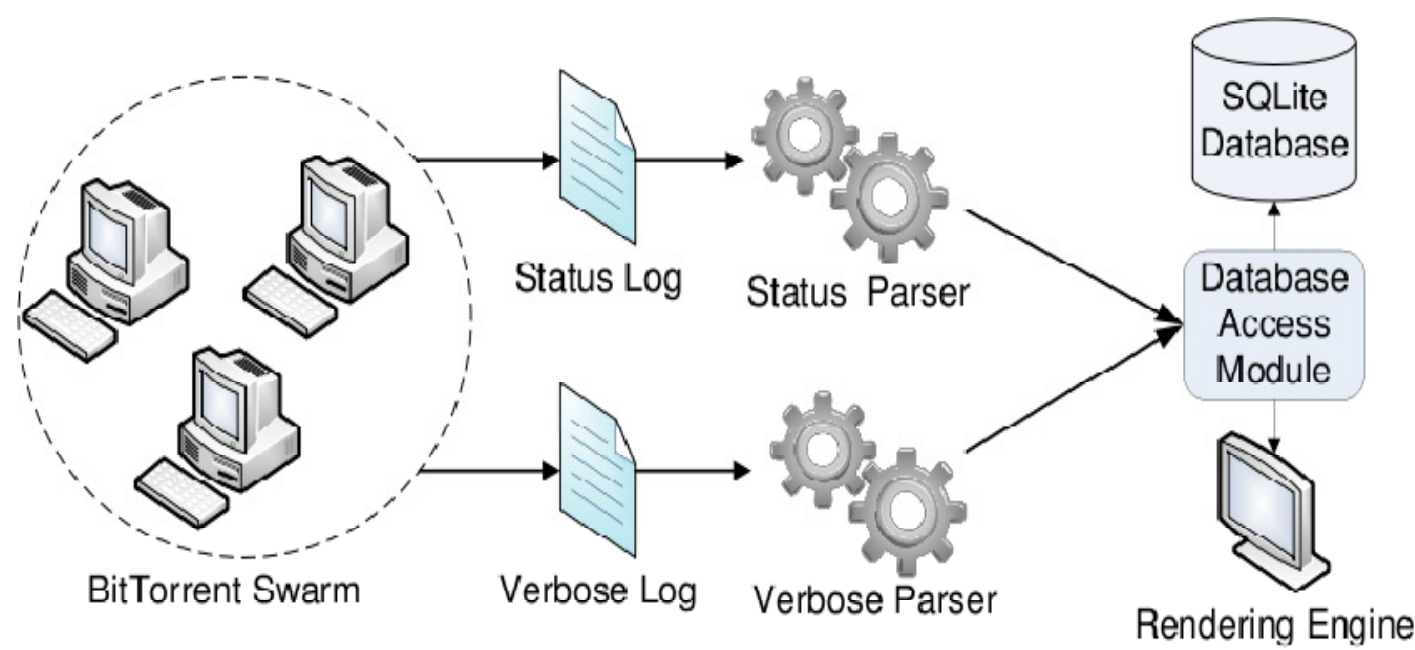

Figure 4. Logging system overview

As shown in figure 4, using parsers specific to each type of logging file, messages are sent as input to the Database Access module that stores them into an SQLite database. In order to analyse peer behaviour the Rendering Engine reads stored logging data using the Database Access module and outputs it to a graphical user interface. More information on each component is presented in the following sections. 
International Journal of Computer Networks \& Communications (IJCNC) Vol.3, No.1, January 2011

\section{A . Physical Infrastucture}

The current setup of the swarm management framework consists of 10 commodity hardware systems (hardware nodes) running 10 OpenVZ virtual environments (VEs), for a each total of 100 virtualized systems. Each virtualized system runs a single Server daemon and a single BitTorrent client.

All hardware nodes are identical with respect to the CPU power, memory capacity and HDD space and are part of the same network. The network connections are 1Gbit Ethernet links. Hardware nodes and virtualized environments are running the same operating system (Debian GNU/Linux Lenny) and the same software configuration.

To simulate real network bandwidth restrictions we usLinux traffic control (the tc tool) or client-centric options to limit peer upload/download speed. As virtualized systems are usually NAT-ed, iptables is also used on the base stations.

As all stations use common scripts and the same BitTorrent clients, important parts of the filesystem are accessed through NFS (Network File System). Thus, in case of 100 virtualized systems, only one of them is actually storing configuration, executable and library files; the other systems use NFS.

Easy system administration has been ensured through the use of cluster-oriented tools such as Cluster SSH or Parallel SSH.

\section{B. XML Configuration Files}

As we wanted to make it as easy as possible to deploy new BitTorrent swarms, we designed our architecture to support two XML configuration files: one for physical nodes configuration and one for BitTorrent swarms configuration.

The modes XML file describes the physical infrastructure configuration. It stores information about:

- $\quad$ physical nodes/OpenVZ containers IP addresses and NATports 1;

- $\quad$ SSH port and username;

- Server and Bittorrent clients paths.

The swarm XML file is used to describe the swarm configuration. It maps a BitTorrent client to a physical node from the nodes XML configuration file, and contains the following information:

- $\quad$ torrent file for the experiment (same path on all containers)

- BitTorrent client upload/download speed limitations.

- $\quad$ output options (download path, logs paths)

The speed limitations are enforced using the tc Linux tool or internal client bandwidth limitation options. 1All the physical machines in the deployed environment are behind NAT.

\section{Commander}

The Commander is a command-line tool that provides easy control over the BitTorrent clients in our experiments by communicating with the Server daemon. It is built entirely in Python and is easily expendable to support new protocol messages and other features.

The Commander receives as input the two XML configuration files discussed in Section IV-B and interacts with the Server through several commands: bootstrap, archive, start, stop, status, getclients, getoutput, cleanup. The bootstrap command is made through SSH and starts the Server daemon(s). The other commands use socket communication to a designated port and specific node IP. Through the Commander, users can send commands to both single or multiple virtualized containers. All commands take as parameters node and client ids.

\section{Server}

The Server application represents a daemon [5] that listens for incoming connections and manages BitTorrent clients. Upon start-up, the server receives as input from the Commander the IP address on 
International Journal of Computer Networks \& Communications (IJCNC) Vol.3, No.1, January 2011

which to bind itself for socket connections. The port on which it listens is predefined in a configuration file visible to both Server and Commander.

Similar to the Commander application, the language chosen for the implementation is Python, which offers several C-like functionalities, like the socket module for communication and the subprocess for process spawning(the server is responsible for starting and stopping the BitTorrent clients). The BitTorrent swarm analysis system described in section II is also entirely implemented in Python, and the Server uses its status file parsers in order to obtain the latest information about a transfer status.

The Server is separated from the BitTorrent clients using a thin layer of classes, implemented for each client, which provide the interface needed for commanding their execution and establishing their input parameters.

\section{COMMUNICATION PROTOCOL}

The system design implies that BitTorrent clients reside on remote machines and are managed through a Server application, which runs as a daemon on their system. This Server is remotely controlled, being started, restarted and stopped using SSH commands initiated through the Commander application. Once the Server is started, the Commander acts as its client, communicating with it in order to control the BitTorrent applications. Our protocol implies that each BitTorrent client started by the Server is associated with only one torrent file.

Currently, the software service infrastructure supports the following messages:

- $\quad$ START-CLIENT - the server will start a client with the given parameters.

- $\quad$ STOP-CLIENT - the server will stop a client with the given identifier.

- GET-CLIENTS - the server replies with a list of running clients.

- GET-OUTPUT - the server replies with information about clients output (running or not)

- ARCHIVE - the server creates archives with the files indicated in the message, and deletes the files.

- $\quad$ GET-STATUS - returns information about an active transfer.

- CLEANUP - removes files, extendable to other file types. The dictionary maps the types of the files that need to be removed, in the current version of the implementation it supports the following keys:

- ALL - if True, then erases all files related to the experiment

- DOWN - if True, erases all downloaded files

- VLOGS - if True, erases all verbose log files

- SLOGS - if True, erases all status log files

- ARCHIVE - if True, erases all archives related the experiment

The Commander initiates transfers by starting a client with a specific torrent file and options (download path, log files paths and names), and the Server returns a corresponding ID, which can be used to check the transfer status. The status information is retrieved from the status log files, and currently supports the following parameters: download speed, upload speed, downloaded size, uploaded size, eta(estimated time of arrival), number of peers. In the reply message body, each parameter uses a string identifier (parameter name) and is followed by its corresponding value.

\section{PROTOCOL MESSAGES AND CLIENT INSTRUMENTATION}

The logging system performs in-depth swarm analysis by inspecting protocol messages exchanged between peers, together with transfer status information such as upload speed, download speed, download percentage, number of peers.

Our study of logging data takes into consideration two opensource BitTorrent applications: Tribler [8] and hrktorrent [6] (based on libtorrent-rasterbar [2]). While the latter needed minimal changes in order to provide the necessary verbose and status data, Tribler had to be modified significantly. 
International Journal of Computer Networks \& Communications (IJCNC) Vol.3, No.1, January 2011

The process of configuring Tribler for logging output is completely automated using shell scripts and may be reversed. The source code alterations are focused on providing both status and verbose messages as client output information.

Status message information provided by Tribler includes transfer completion percentage, download and upload rates. In the modified version, it also outputs current date and time, transfer size, estimated time of arrival (ETA), number of peers, and the name and path of the transferred file.

In order to enable verbose message output, we took advantage of the fact that Tribler uses flags that can trigger printing to standard output for various implementation details, among which are the actions related to receiving and sending BitTorrent messages. The files we identified to be responsible for protocol data are changed using scripts in order to print the necessary information and to associate it to a timestamp and date. Since most of the protocol exchange data was passed through several levels in Tribler's class hierarchy, attention had to be paid to avoid duplicate output and to reduce file size. In contrast to libtorrent-rasterbar, which, at each transfer, creates a separate session log file for each peer, Tribler stores verbose messages in a single file. This file is passed to the verbose parser, which extracts relevant parts of the messages and writes them into the database.

Unlike Tribler, hrktorrent's instrumentation did not imply modifying its source code but defining TORRENT_LOGGING and TORRENT_VERBOSE_LOGGING macros before building (recompiling) libtorrent-rasterbar. Minor updates had to be delivered to the compile options of hrktorrent in order to enable logging output.

The BitTorrent clients and log parsers are configured to distinguish between the following protocol messages [1]:

- choke and unchoke - notification that no data will be sent until unchoking happens.

- interested and not interested - notifies of a peer's 'interested'/'uninterested' state2. Data transfer takes place whenever one side is interested and the other side is not choking.

- have - sent to inform all peers of a piece's successful download (its hash matches the one from the .torrent metafile)3.

- $\quad$ bitfield - sent after an initial handshaking sequence between peers. The payload is a bitfield representing the pieces that have been successfully downloaded.

- request - sent to obtain blocks of data, the payload contains a piece index and the block's length and offset within the piece.

- $\quad$ piece - contains a block of data, its position within a piece and the piece's index. By default these messages are correlated with request messages, but there are cases when an unexpected piece arrives if choke and unchoke messages are sent in quick succession and/or transfer is going very slowly.

- cancel - cancels a request for a piece; it has the same payload as the request message. These messages are commonly used when the download is almost complete; request messages are sent to many peers to make sure the final pieces arrive quickly; when a piece is downloaded its other requests are cancelled.

Although our system processes and stores all protocol message types, the most important messages for our swarm analysis are those related to changing a peer's state (choke/unchoke) and requesting/receiving data. Correlations between these messages are the heart of provisioning information about the peers' behaviour and BitTorrent clients' performance.

${ }^{2}$ Connections contain two bits of state on either end: choked or not, and interested or not.

${ }^{3}$ The peer protocol refers to pieces of the file by index as described in the metainfo file (.torrent file), starting at zero. Connections contain two bits of state on either end - choked or not, and interested or not. 
International Journal of Computer Networks \& Communications (IJCNC) Vol.3, No.1, January 2011

\section{STORAGE ENGINE}

The swarm analysis infrastructure contains of two levels of storage:

- $\quad$ status and verbose log files output by clients and sent to parser modules,

- database storage populated by parser modules and used by the rendering interface.

Log files are created during a running experiment and parsed after the experiment had completed. Parsed data is collected as offline information. All information is subsequently stored in a database file.

The database storage module enables persistence and rapid searching of relevant information, stored as status data and verbose data. All experiment data is stored in a single SQLite database file that allows easy migration and copying.

The storage engine represents an efficient method for collecting information, compared to using XML files or other filebased approaches. For example, a 5.8 GB worth of text file containing verbose logs was parsed and stored in a database file of $518 \mathrm{MB}$.

In addition to holding logging messages, the database stores properties of BitTorrent clients and details about the swarm (number of peers, number of initial seeders, start time, file name, file size). It also stores hardware characteristics about the machine it is running on, such as CPU description, RAM size, operating system version and network specific information. Along with these, transfer speed limitations (ifany) are stored for each client.

A thin Python layer allows access to the parser and rendering engine for writing and reading, respectively, data to/from the database. Sample queries include adding/deleting a new peer, adding/deleting a verbose message (a BitTorrent protocol [1] message), listing messages for a given client in a specific time frame, listing certain types of BitTorrent messages. In the current infrastructure, the rendering engine acts as a presentation layer for collected information.

\section{RESULT PROCESSING}

Once all logging and verbose data from a given experiment is collected, the next step is the analysis phase. The testing infrastructure provides a GUI (Graphical User Interface) statistics engine for inspecting peer behaviour.

The GUI is implemented in Python using two libraries: matplotlib - for generating graphs and TraitsUi - for handling widgets. It offers several important plotting options for describing peer behaviour and peer interaction during the experiment:

- $\quad$ download/upload speed - displays the evolution of download/ upload speed for the peer;

- $\quad$ acceleration - shows how fast the download/upload speed of the peer increases/decreases;

- $\quad$ statistics - displays the types and amount of verbose messages the peer exchanged with other peers.

The last two options are important as they provide valuable information about the performance of the BitTorrent client and how this performance is influenced by protocol messages exchanged by the client.

The acceleration option measures how fast a BitTorrent client is able to download data. High acceleration forms a basic requirement in live streaming, as it means starting playback of a torrent file with little delay.

The statistics option displays the flow of protocol messages. As stated in Section VI, we are interested in the choke/unchoke messages. The GUI also offers two modes of operation: "Single Client Mode", in which the user can follow the behaviour of a single peer during a given experiment, and "Client Comparison Mode", allowing for comparisons between two peers. 
International Journal of Computer Networks \& Communications (IJCNC) Vol.3, No.1, January 2011

\section{EXPERIMENTAL RESULTS}

\section{A .Experimental Setup}

As stated in II, the software service infrastructure allows BitTorrent swarm management. The current implementation was tested on several scenarios for three clients: Hrktorrent, Tribler and Transmission. The experiments were conducted on the physical infrastructure presented in IV-A and involved checking all the functionalities provided by the services. The swarms created during these scenarios provided tens of GB of logging data for the analysis system.

The current setup of our testing infrastructure consists of 10 commodity hardware systems (hardware nodes) each running 10 OpenVZ virtual environments (VEs), for a total of 100 virtualized systems. Each virtualized system runs a single BitTorrent peer.

All hardware nodes are identical with respect to the CPU power, memory capacity and HDD space and are part of the same network. The network connections are 1Gbit Ethernet links. Hardware nodes and virtualized environments are running he same operating system (Debian GNU/Linux Lenny) and the same software configuration.

To simulate real network bandwidth restrictions we used Linux traffic control (the tc tool) to limit peer upload/ download speed.

\section{B. Results and Measurements}

All our experiments have taken place in a controlled environment or closed swarm. As such we have had complete control over the peers and the network topology, allowing us to define the constraints of each scenario.

We ran several download sessions using the libtorrent/ hrktorrent and Tribler BitTorrent clients and files of different sizes. All scenarios involved simultaneous downloads for all clients. At the end of each session, download status information and extensive logging and debugging information were gathered from each client.

The experiments made use of all 100 virtualized peers which were configured to use bandwidth limitations. Half of the peers (50) were considered to be high-bandwidth peers, while the other half were considered to be low-bandwidth peers. The high-bandwidth peers were limited to $512 \mathrm{~KB} / \mathrm{s}$ download speed and $256 \mathrm{~KB} / \mathrm{s}$ upload speed and the low-bandwidth peers were limited to $64 \mathrm{~KB} / \mathrm{s}$ download speed and $32 \mathrm{~KB} / \mathrm{s}$ upload speed.

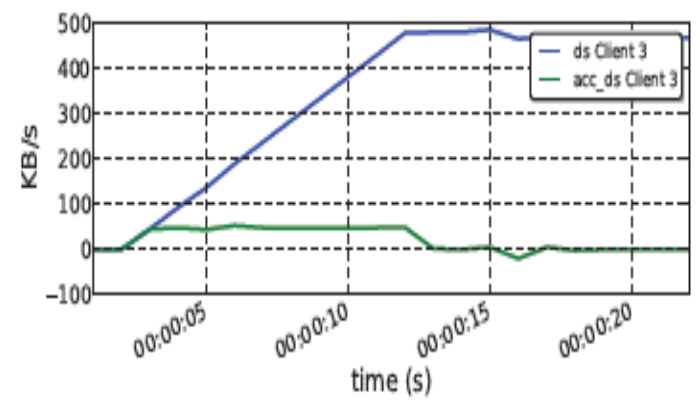

Figure 5. Download speed/acceleration evolution (libtorrent BitTorrent client) 
International Journal of Computer Networks \& Communications (IJCNC) Vol.3, No.1, January 2011

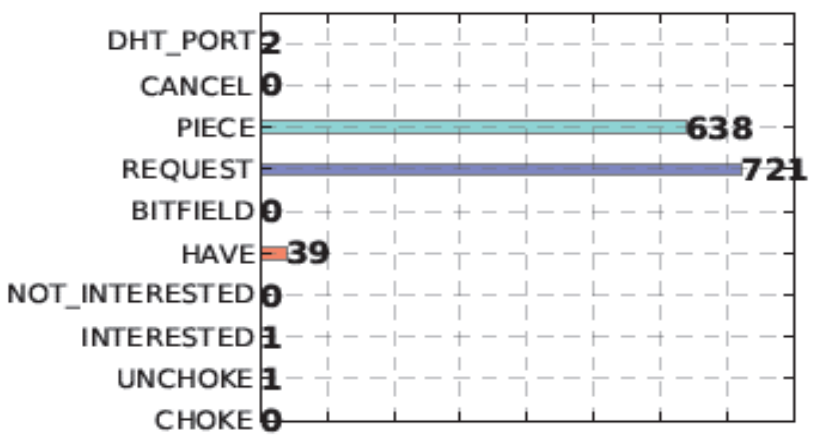

Figure 6. BitTorrent protocol messages (20 seconds)

Figure 5 displays a 20 seconds time-based evolution of the download speed and acceleration of a peer running the libtorrent client. Acceleration is high during the first 12 seconds, when a peer reaches its maximum download speed of around 512KB/s. Afterwards, the peer's download speed is stabilized and its acceleration is close to 0 .

All non-seeder peers display a similar start-up pattern. There is an initial 10-12 seconds bootstrap phase with high acceleration and rapid reach of its download limit, and a stable phase with the acceleration close to 0 .

Figure 6 displays messages exchanged during the first 20seconds of a peer's download session, in direct connection with Figure 5. The peer is quite aggressive in its bootstrap phase and manages to request and receive a high number of pieces. Almost all requests sent were replied with a block of data from a piece of the file.

The download speed/acceleration time-based evolution graph and the protocol messages numbering are usually correlated and allow detailed analysis of a peer's behaviour. Our goal is to use this information to discover weak spots and areas to be improved in a given implementation or swarm or network topology.

\section{RELATED WORK}

As BitTorrent has become the most heavily used peer-topeer protocol in the Internet, there have been many measurement studies related to its internals, enhancements and swarm entities.

Most measurements and evaluations involving the BitTorrent protocol and applications are either concerned with the behavior of a real-world swarm or with the internal design of the protocol. There has been little focus on creating a self-sustained swarm management environment capable of deploying hundreds of controlled peers, and subsequently gathering results and interpreting them.

The PlanetLab infrastructure provides a realistic testbed for Peer-to-Peer experiments. PlanetLab nodes are connected to the Internet and experiments have a more realistic testbed where delays, bandwidth and other are subject to change. Tools are also available to aid in conducting experiments and data collection.

A testing environment involving four major BitTorrent trackers for measuring topology and path characteristics has been deployed by Iosup et al. [14]. They used nodes in PlanetLab. The measurements were focused on geo-location and required access to a set of nodes in PlanetLab.

Dragos Ilie et al. [13] developed a measurement infrastructure with the purpose of analyzing P2P traffic. The measurement methodology is based on using application logging and link-layer packet capture.

One notable study related to BitTorrent protocol analysis is [15]. The authors' efforts are directed towards correlating characteristics of BitTorrent and its Internet underlay, with focus on topology, connectivity, and path-specific properties. For this purpose they designed and implemented Multiprobe, a framework for large-scale P2P file sharing measurements. The main difference between their implementation and our approach is that we focus on an in-depth client-level analysis and not on the whole swarm. 
International Journal of Computer Networks \& Communications (IJCNC) Vol.3, No.1, January 2011

In [16] Meulpolder et al. present a mathematical model for bandwidth-inhomogeneous BitTorrent swarms. Based on a detailed analysis of BitTorrent's unchoke policy for both seeders and leechers, they study the dynamics of peers with different bandwidths, monitoring their unchoking and uploading/ downloading behavior. Their analysis showed that having only peers with the same bandwidth is not enough to determine in-depth the peers' behavior. In those experiments they split the peers into two bandwidth classes - slow and fast - and they observed that slow ones usually unchoked other slow peers, their data being transfered from fast peers. Although they do not offer precise details about the experimental part of monitoring unchoking behavior and transfers rates, their work relates to what we intend to do with the logging messages that our system parses and stores.

While [16] provides a peer level analysis, another approach is to study BitTorrent at tracker level, as described in [9]. This paper implements a scalable and extensible BitTorrent tracker monitoring architecture, currently used in the Ubuntu Torrent Experiment[4] experiment at University Politehnica of Bucharest, the Computer Science and Engineering Department. The system analyses the peer-to-peer network considering both the statistic data variation and the geographical distribution of data. This study is based on a similar infrastructure with the one we use for our client and protocol level analysis.

\section{CONCLUSION AND FURTHER WORK}

The client-side detailed analysis approach presented earlier is used for evaluating peer-to-peer swarms and BitTorrent implementations. We have designed and implemented a message collection and visualisation facility that allows indepth analysis of protocol implementations and enhancements. Several experiments were conducted resulting in large amount of collected data that were parsed, stored and subjected to analysis through a GUI statistics engine.

Peer-to-peer measurement infrastructures are commonly using tracker information or probe-based information, offering an overall view of a swarm. While not as scalable, our approach allows collection of in depth data such as lowlevel protocol information and verbose logging messages. This requires control of swarm peers, resulting in closed/controlled swarms providing full information and open/external swarms providing partial information.

The infrastructure consists of virtualized commodity hardware systems, instrumented clients that provide extensive information, message parsing modules, a storage engine and a GUI statistics and interpretation engine. It allows comparisons between different protocol implementations and studying the impact of swarm and network characteristics on peer behavior and overall swarm performance.

The framework is a service-based infrastructure intended to be used in conjunction with a result interpretation framework, which collects relevant information from deployed scenarios and uses that information for analysis and dissemination.

The advantages of the framework are automation, high degree of control and access to client logging information regarding protocol internals and transfer evolution. Realistic scenarios can be deployed and monitored, resulting in important information provided by client to be subject of subsequent analysis.

As of this point, the framework has been used for internal scenarios. The goal is to provide the complete infrastructure as a service to be used for running a wide variety of scenarios. We intend to add scheduling options that allow users to plan their experiments to be run at a certain time in the future when enough peers are available.

Traffic shaping is ensured statically at the beginning of each session. We plan to add a dynamic bandwidth shaping facility that would allow altering available bandwidth as if there were other communication sessions on the same link. In order to minimize the administrative configuration, one of the objectives is to use Linux bridging and connect all virtualized systems together without the need for NAT.

In order to improve usability, an important objective is to add a web-based interface to the Commander, which is currently a CLI program. This would provide the advantage of easy access and configuration of the swarm management framework.

Currently, the infrastructure supports the hrktorrent [6] and Tribler [8] implementations. We plan to add support for other popular open-source clients such as Transmission and Vuze. The open-source condition is required as client instrumentation is needed to provide in-depth information. 
International Journal of Computer Networks \& Communications (IJCNC) Vol.3, No.1, January 2011

Extensive simulation and testing and result processing form the major aims of future planning. We plan to design and run a wide variety of test scenarios that will result in large amounts of information to be processed and analysed. Scenarios will focus on measuring the impact of swarm characteristics on peer behaviour, peer performance and overall swarm performance.Our current experiments take into account network characteristics such as bandwidth limitations and swarm characteristics such as client type, client startup time. We plan to extend these and include the impact of NAT and firewalled peers, DHT, PEX, peer localisation, network topology, churning, etc.

As client instrumentation provides in-depth information on client implementation, it generates extensive input for result analysis. Coupled with carefully crafted experiments and message filtering, this will allow the detection of weak spots and of improvement possibilities in current implementations. Thus it will provide feedback to client and protocol implementations and swarm "tuning" suggestions, which in turn will enable high performance swarms and rapid content delivery in peer -to- peer systems.

\section{ACKNOWLEDGMENT}

The authors would like to thank Alex Heris, anu for kindly providing access to the NCIT cluster systems we have been using throughout our experiments.

Special thanks go the the P2P-Next [3] team who is working enthusiastically to deliver the next generation peer-to-peer content delivery platform. Their dedication, professionalism and vision are a constant factor of motivation and focus for our work.

\section{REFERENCES}

[1] BitTorrent Official Specification. http://bittorrent.org/beps/bep -0003.html.

[2] libtorrent (Rasterbar). http://www.rasterbar.com/products/libtorrent/.

[3] P2P-Next. http://www.p2p-next.org/.

[4] Ubuntu Torrent Experiment. http://torrent.cs.pub.ro/.

[5] Daemon behaviour. http://www.python.org/dev/peps/pep-3143/correctdaemon-behaviour, Last accessed on June 30th, 2010.

[6] hrktorrent. http://50hz.ws/hrktorrent/, Last accessed on June 30th, 2010.

[7] ipoque Internet Studies. http://www.ipoque.com/resources/internetstudies/internet-study-2008 2009, Last accessed on June 30th, 2010.

[8] Tribler. http://www.tribler.org/trac/wiki, Last accessed on June 30th,2010.

[9] M. Bardac, G. Milescu, and R. Deaconescu. Monitoring a BitTorrent Tracker for Peer-to-Peer System Analysis. IntelligentDistributed Computing, pages 203-208, 2009.

[10] R. Deaconescu, G. Milescu, B. Aurelian, R. Rughinis, , and N. T, uapus, . A Virtualized Infrastructure for Automated BitTorrent Performance Testing and Evaluation. International Journal on Advances in Systems and Measurements, 2(2\&3):236-247, 2009.

[11] R. Deaconescu, R. Rughinis, , and N. T, `apus, . A Virtualized Testing Environment for BitTorrent Applications. Proceedings of CSCS'17, 2009.

[12] R. Deaconescu, M. Sandu-Popa, A. Draaghici, and N. T, ־apus, . Using Enhanced Logging for BitTorrent Swarm Analysis. Proceedings of $9^{\text {th }}$ RoEduNet International Conference, 2010.

[13] D. Ilie, D. Erman, A. Popescu, and A. Nilsson. Traffic Measurements of P2P Systems. 2004.

[14] A. Iosup, P. Garbacki, J. Pouwelse, and D. Epema. Correlating Topology and Path Characteristics of Overlay Networks and the Internet. CCGRID, 2006.

[15] A. Iosup, P. Garbacki, J. A. Pouwelse, and D. H. Epema. Correlating Topology and Path Characteristics of Overlay Networks and the Internet. October 2005.

[16] M. Meulpolder, J. Pouwelse, D. Epema, and H. Sips. Modeling and Analysis of BandwidthInhomogeneous Swarms in BitTorrent. Proc. Of IEEE P2P 2009, pages 232 - 241, 2009. 